\section{Medication Therapy Management Services for Long-term Care Patients: No Road Maps for Those Trying to Find Their Way}

As required by the Medicare Modernization Act, ${ }^{1}$ organizations offering Medicare Part D prescription drug plans (PDPs) are obligated to provide medication therapy management programs (MTMPs) as part of the benefit. While medication therapy management (MTM) has been performed by pharmacists in a variety of settings for years, a consensus definition of MTM was not developed until last year. ${ }^{2}$ As defined in the Act, MTMPs are "furnished by a pharmacist [and] designed to assure ... that covered part D drugs are appropriately used to optimize therapeutic outcomes through improved medication use, and to reduce the risk of adverse events, including adverse drug interactions."

While the Act defines the beneficiaries who are targeted for MTM (those with multiple chronic conditions, taking multiple medications, and likely to have high drug expenses) and basic elements of the program (promotion of enhanced enrollee understanding of the appropriate use of medications, adverse event risk reduction, increased medication adherence, and detection of adverse drug events and patterns of prescription drug overuse or underuse), the government has taken a decidedly hands-off approach to regulating MTMPs. Citing insufficient standards and performance measures to evaluate MTMPs and the inability to determine what requirements would enhance MTMPs and improve patient outcomes, the Centers for Medicare and Medicaid Services (CMS) has given what it considers maximum flexibility to plans so they may develop MTMPs that can achieve the goal of improving therapeutic outcomes.

Organizations that bid on the Part D drug plans have remained silent about their MTMP plans, but some guidance is available as to what can be expected for MTM services at certain pharmacy settings. In April 2005, the American Pharmacists Association and the National Association of Chain Drug Stores Foundation released a model framework for implementing MTM services in a community pharmacy setting. ${ }^{3}$ The framework (a) provides 9 factors that community pharmacists can consider when targeting patients for MTM services, (b) identifies 5 core components of MTM programs in community pharmacy (medication therapy review, a personal medication record, a medication action plan, intervention and referral, and documentation and follow-up) and describes pharmacist responsibilities for each, and (c) provides sample personal medication records and medication action plans for community pharmacists to use in their programs. Regarding senior care, the American Society of Consultant Pharmacists (ASCP) released an issue paper on MTM services for ambulatory Medicare beneficiaries in April 2004, reviewing the goals of MTM services in the elderly and the types of MTM services that a pharmacist could provide to ambulatory seniors. ${ }^{4}$ An MTMP for low-income ambulatory seniors was described by Stebbins et al. in a recent issue of JMCP. ${ }^{5}$
However, there is little guidance for developing MTM services for those residing in long-term care (LTC) facilities. At this time, the only point that is clear is that MTM services need to be distinct from the monthly drug regimen review (DRR) process mandated for all LTC residents. While the DRR process may save as much as $\$ 3.6$ billion per year in medication-related problems, these problems still generate an estimated $\$ 4$ billion in annual costs in the LTC setting. ${ }^{6}$ Inappropriate prescribing remains a problem for the nation's 1.8 million LTC and assistedliving residents and is a significant factor contributing to the risks for morbidity and mortality ${ }^{7-9}$

While the deadline has passed for submitting bids for Part D PDPs for 2006, organizations preparing bids for PDPs for 2007 and beyond should look for evidence of success in MTM services in the LTC arena when formulating their bids. In this issue, Trygstad et al. describe results of the North Carolina Polypharmacy Initiative, a targeted drug therapy management consultation where a pharmacist reviews medical records and Medicaid pharmacy claim drug profiles to determine if a potential drug therapy problem alert requires action and, if so, to make interventions and determine if a therapy change was enacted. ${ }^{10}$ As measured by the study objectives, the program was successful because it was associated with a reduction in potential drug therapy problem alerts and median per-patient-per-month prescription drug costs. The program further demonstrated the utility of consultant pharmacists in the LTC setting since physicians implemented nearly $60 \%$ of pharmacist recommendations.

However, the study by Trygstad et al. did not look at health outcomes and therefore did not determine if the drug therapy changes would maintain or improve the quality of care that residents received. It is unlikely that a consultant pharmacist would make a recommendation that would result in lower quality of care for an LTC resident, but the study by Trygstad et al. measured only intermediate (process of care) and drug cost outcomes. Research is still necessary to demonstrate that a pharmacy intervention program designed for multiple disease states in LTC leads to improvements in resident health outcomes. Such research would provide powerful evidence of an intervention's utility. It is easy to sympathize with the researchers; by intervening in many different disease states, it is very difficult to quantify the impact of the program on LTC resident health outcomes. Limiting the study to retrospective pharmacy claims data makes even more remote the possibility of determining health outcomes.

In the MTMP described in the study, the pharmacist had the ability to recommend that drug therapy be withdrawn, modified, or added, as each case required. Changes to drug therapy impact more than prescription drug expenses; these changes can have an effect on immediate and longer-term medical expenses as well. However, the study only presented prescription drug costs and did not report the impact of the intervention on medical costs or overall health care costs. The true cost for a 
health system of an intervention or series of interventions is best assessed by examining overall costs.

How Part D providers choose to examine the costs of MTMPs may very well depend upon the type of program they are offering. Medicare Advantage prescription drug (MA-PD) plans are at risk for overall health costs, so their level of interest in the impact of a MTMP on overall costs is likely to be greater than a PDP, which is not at risk for overall health costs. One must wonder if the Part D program will suffer from the silo approach to health care finance that managed care plans have struggled to overcome. CMS's proposal ${ }^{11}$ for linking deidentified Part D prescription claim data to hospital, physician, and other medical utilization data in a public database may allow health policy analysts to compare prescription drug expenses and overall health care expenses for PDPs and MA-PDs.

More questions than answers presently remain pertaining to MTM services in LTC facilities. While the forthcoming report from the Department of Health and Human Services on standards of practice for pharmacy services (including clinical services) provided to patients in LTC settings will be a useful tool for PDP developers to consider when designing or refining MTMPs, the experiences that the LTC industry encounter starting on January 1, 2006 ("Part D-Day"), will be even more valuable. The sharing of MTM best practices by PDPs and LTC facilities can reduce the trial-and-error approach to MTM services and can improve the quality of services delivered to LTC residents.

Joshua J. Spooner, PharmD, MS Director, Clinical and Outcomes Services Advanced Concepts Institute University of the Sciences in Philadelphia Philadelphia, Pennsylvania j.spoone@usip.edu

\section{DISCLOSURE}

The author discloses no potential bias or conflict of interest relating to this editorial.

\section{REFERENCES}

1. Public Law 108-173. The Medicare Prescription Drug, Improvement, and Modernization Act of 2003. December 8, 2003. Available at: http://www.cms. hhs.gov/medicarereform/. Accessed August 12, 2005.

2. Medication therapy management services definition and program criteria. July 27, 2004. Available at: http://www.amcp.org/data/jmcp/Letters-179186.pdf and http://www.aacp.org/Docs/MainNavigation/Resources/ 6308_MTMServicesDefinitionandProgramCriteria27-Jul-04.pdf. Accessed August 12, 2005.

3. The American Pharmacists Association and the National Association of Chain Drug Stores Foundation. Medication therapy management in community pharmacy practice: core elements of an MTM service. Version 1.0. April 29, 2005. Available at: http://www.aphanet.org/AM/Template.cfm?Template=/ CM/ContentDisplay.cfm\&ContentID=3303. Accessed August 12, 2005.

4. The American Society of Consultant Pharmacists. Medication therapy management services for ambulatory Medicare beneficiaries. April 28, 2004. Available at: http://www.ascp.com/medicarerx/docs/ASCPMTMS.pdf. Accessed August 12, 2005.

5. Stebbins MR, Kaufman DJ, Levens Lipton H. The PRICE clinic for lowincome elderly: a managed care model for implementing pharmacist-directed services. J Manag Care Pharm. 2005;11(4):333-41.

6. Bootman JL, Harrison DL, Cox E. The health care cost of drug-related morbidity and mortality in nursing facilities. Arch Intern Med. 1997;157:2089-96.

7. Gurwitz JH, Field TS, Avorn J, et al. Incidence and preventability of adverse drug events in the nursing home setting. Am J Med. 2000;109:87-94.

8. Sloane PD, Gruber-Baldini AL, Zimmerman S, et al. Medication undertreatment in assisted living settings. Arch Intern Med. 2004;164:1621-25.

9. Gerety MB, Cornell JE, Plichta DT, Eimer M. Adverse events related to drugs and drug withdrawal in nursing home residents. J Am Geriatr Soc. 1993:41:1326-32.

10. Trygstad TK, Christensen D, Garmise J, Sullivan R, Wegner SE. Pharmacist response to alerts generated from Medicaid pharmacy claims in a long-term care setting: results from the North Carolina Polypharmacy Initiative. J Manag Care Pharm. 2005;11(7):575-83.

11. Centers for Medicare and Medicaid Services. Medicare prescription drug data strategy: improving evidence for patient care through the Medicare prescription drug benefit. Available at: http://www.cms.hhs.gov/medicarereform/ CMSPaper-DataStrategyforMedicareDrugBenefitOverview.pdf. Accessed August 8,2005 . 